\title{
Evaluación de la producción limpia de papa criolla (Solanum phureja) en Madrid, Cundinamarca
}

\author{
Maikol Santamaría G. \\ Johana Montañéz $G$. \\ Rafael Ricardo Sánchez P.
}

Recibido el 10 de junio de 2010. Aprobado el 12 de octubre de 2010

\begin{abstract}
Resumen
El presente artículo, muestra los resultados del estudio que consistió en evaluar la producción limpia y convencional de papa criolla (Solanum phureja) en el Centro Agroecológico de Investigación y Capacitación (Ceagro) San Pablo, Madria Cundinamarca. Se implementó para la producción limpia, fertilización orgánica comercial y manejo fitosanitario con insumos biológicos; y para la producción convencional fertilización orgánica comercial mas abono mineral compuesto 10-30-10 y manejo fitosanitario con insumos de síntesis química. Los resultados mostraron que en las variables evaluadas número de tubérculos por planta, tamaño y peso de tubérculos por planta y peso de la producción por planta no se presentaron diferencias estadísticamente significativas entre tratamientos. Estos resultados sugieren que en las condiciones del Ceagro San Pablo se pueden obtener producciones de Solanum phureja mediante alternativas de manejo limpias que no deterioran el ambiente y mejoran la calidad de los tubérculos.
\end{abstract}

\section{Palabras clave}

Fertilización, manejo fitosanitario, agricultura limpia, agroecología.

\section{Abstract}

The present work consisted of evaluating the clean and conventional agriculture of native potato (Solanum phureja) in the Research and Training Agroecological Center (Ceagro) San Pablo. Was implemented for clean production commercial organic fertilization and fitosanitary management with biological products; and for conventional production fertilization with commercial organic fertilization more mineral fertilizer 10-30-10 and fitosanitary management with chemical products. The results showed that in the variables evaluated number of tubers for plant, size and weight of tubercles for plant and weight of the production for plant, did not presented statistically significant differences between treatments. These results suggest that conditions of Ceagro San Pablo can obtained productions of Solanum phureja by clean alternatives management, that do not deteriorate the environment and improve the quality of tubers

\section{Keywords}

Fertilization, fitosanitary management, clean agriculture, agroecology. 


\section{Introducción}

El cultivo de papa criolla es uno de los sistemas productivos más importantes económica y socialmente en Colombia ya que representa entre el 5 y $10 \%$ de la papa cultivada del país y su exportación asciende a mil toneladas al año (Ministerio de Agricultura y desarrollo Rural, 2008; Agencia de Noticias de Ciencia y Tecnología de Colombia, 2005). Según el Ministerio de Agricultura, en el año 2008 se sembraron cerca de 160.000 hectáreas de papa con un rendimiento promedio de 17.8 ton/ha. En el departamento de Cundinamarca, el área sembrada en el año 2008 alcanzó 60.000 hectáreas con un rendimiento promedio de 18 ton/ha. En Colombia, dicho sistema productivo es de gran importancia debido a que es desarrollado por aproximadamente 90.000 familias (Cevipapa, 2004).

Para el establecimiento de este cultivo se realiza una preparación excesiva del suelo, uso indiscriminado de insumos de síntesis química y fertilización sin considerar el balance de nutrientes causando el deterioro de los suelos (Muñoz L. y Lucero M., 2008). En el ciclo productivo de papa criolla la fertilización y el manejo fitosanitario son los rubros con mayor incidencia dentro de los costos de producción, participando con el $27 \%$ y $18.2 \%$ respectivamente (MADR, 2009) manejando dosis de fertilizantes que superan los $900 \mathrm{~kg} / \mathrm{ha}$ con crecimiento vegetativo exuberante y reducción de la producción (Muñoz L. y Lucero M., 2008).

La papa es el producto de origen agrícola que demanda mayor cantidad de insecticidas y fungicidas y el segundo de fertilizantes después del café (Buitrago et al., 2004). Varios estudios en Colombia han evidenciado el papel de los elementos mayores y menores en los rendimientos de la papa criolla (Pérez et al., 2008; Muñoz L. y Lucero M., 2008; Arias, 1996). Muñoz y Lucero (2008) evaluaron el efecto de la fertilización orgánica en la papa criolla en el departamento de Nariño obteniendo buenos resultados con la mezcla $300-800 \mathrm{~kg} / \mathrm{ha}$ de fertilizante químico 13-26-6 y abono orgánico respectivamente, mejorando la relación costo beneficio.

El manejo fitosanitario O MIP (Manejo Integrado de Plagas) actualmente incluye estrategias culturales, químicas y biológicas que generalmente no se implementan de forma técnica y sin tener en cuenta la incidencia de las plagas y enfermedades a través del desarrollo del

cultivo, con aplicaciones tipo calendario de insumos altamente tóxicos que perjudican económicamente al productor al generarle dependencia de éstos y efectos nocivos al ambiente. El manejo cultural y biológico de plagas en papa como nuevas alternativas de producción, esta constituido por el uso de semilla certificada, labranza mínima, recolección de residuos, uso de entomopatógenos, trampas, densidades de siembra, rotación de cultivos entre otras (CIP, 1992; Fedepapa, 2005).

Lo anterior, evidencia la necesidad de evaluar alternativas de manejo agroecológico de cultivos de papa criolla y sus ventajas frente al manejo convencional, teniendo en cuenta las limitantes agroclimatológicas que para este fin presenta el ambiente de la Sabana de Bogotá y la dependencia de los cultivadores hacia tecnologías convencionales de manejo. El presente trabajo tuvo como objetivo evaluar el número de tubérculos producidos por planta de papa criolla, el tamaño y el peso de éstos con la implementación de fertilización y el manejo fitosanitario limpio y convencional.

\section{Materiales y Métodos}

\section{Localización}

El trabajo de investigación se realizó en el Centro Agroecológico de Investigación y Capacitación Ceagro San Pablo, ubicado en el Municipio de Madrid Cundinamarca que cuenta con una temperatura media registrada de $14^{\circ} \mathrm{C}$; precipitación promedio de 598 mm anual (región seca), con índice de aridez de 10.3 y un índice hídrico de 6.2. Las máximas temporadas de lluvias se presentan entre los meses de abril - mayo y octubre - noviembre, las cuales no son constantes en el tiempo, debido a los efectos del cambio climático.

\section{Diseño Experimental}

Se sembraron tubérculos de papa criolla pertenecientes a la variedad "yema de huevo", provenientes de cultivos productores de semilla del municipio de

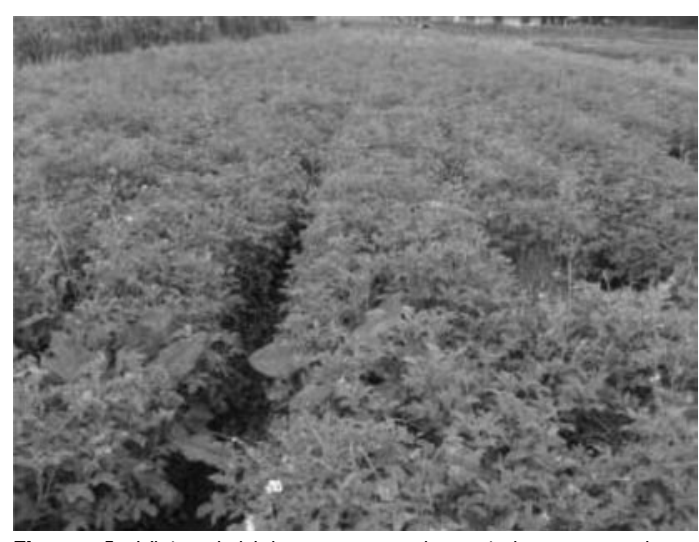

Figura 1. Vista del bloque experimental uno en pleno desarrollo. Fuente: Los autores. 2009. Sibaté Cundinamarca. El lote seleccionado se dividió en tres bloques de $10 \mathrm{~m}$ x $60 \mathrm{~m}$ cada uno, para un área total de 600 $\mathrm{m}^{2}$ por bloque, con separación de $6 \mathrm{~m}$ entre ellos (Figura 1). Cada bloque se dividió en dos parcelas de $10 \mathrm{~m} \times 25 \mathrm{~m}$ con separación de $10 \mathrm{~m}$ entre ellas. En cada parcela se trazaron 10 surcos y se sembraron entre uno y dos tubérculos cada $30 \mathrm{~cm}$. El área total del ensayo y la unidad experimental fue de 2520 $\mathrm{m}^{2}$ y $250 \mathrm{~m}^{2}$, respectivamente. 
El diseño estadístico para las variables evaluadas correspondió a un diseño de bloques al azar. A los datos obtenidos se les realizó análisis de varianza y fueron sometidos a un estadístico de prueba LSD (diferencia mínima significativa) con 95\% de confiabilidad; por medio del programa STATGRAPHICS Plus.

\section{Fertilización}

En ambos tratamientos, limpio y convencional, la fertilización se fraccionó en dos aplicaciones: en el momento de la siembra y a los 45 días en el momento del aporque. En el tratamiento de producción limpia se aplicó gallinaza y humus comercial en una relación 1:1. En el tratamiento de producción convencional se aplicó abono mineral compuesto 1030-10 mas gallinaza y humus en una relación 0.5:1:1 respectivamente (Figura 2).

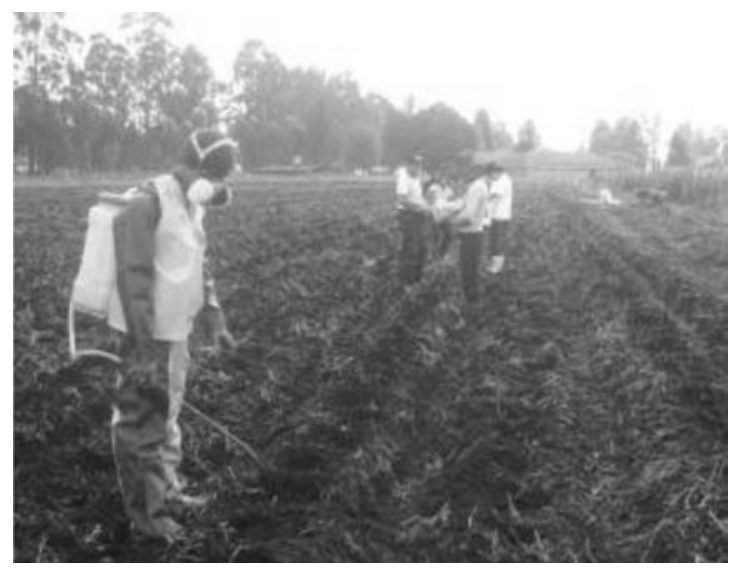

Figura 2. Siembra y aplicación de insumos en los bloques experimentales. Fuente: Los autores. 2009

\section{Manejo fitosanitario}

En los tratamientos limpio y convencional las medidas de control de riesgos fitosanitarios iniciaron desde la siembra. En el tratamiento limpio se utilizó para el manejo de plagas y enfermedades azadirachtina (extracto de nemm), sales de potasio, extracto de ajenjo, carbonato de cobre y sulfato de calcio (caldo bordeles), Beauveria bassiana, Trichoderma harzianum, y oxicloruro de cobre. En el tratamiento convencional se utilizó metaldehído (molusquicida comercial), carbofuran 45\% (Furadán), azadirachtina (extracto de nemm), oxicloruro de cobre y mancozeb. Las aplicaciones de los insumos en ambos tratamientos se realizaron teniendo en cuenta muestreos semanales de plagas y enfermedades.

\section{Variables evaluadas}

Número de tubérculos por planta: Al momento de la cosecha se seleccionaron 20 plantas al azar por repetición de cada tratamiento y se contó el número de tubérculos con diámetro no menor a $3 \mathrm{~cm}$ (características comerciales de primera, segunda o tercera).
Tamaño y peso por tubérculo: En cosecha se midió el diámetro y el peso de 20 tubérculos al azar por planta seleccionada que tuvieran características comerciales (primera, segunda o tercera).

Peso de la producción por planta: Consistió en pesar el total de tubérculos, con características comerciales, obtenidos en cada una de las 20 plantas seleccionadas.

\section{Resultados y Discusión}

Los datos obtenidos se examinaron mediante análisis de varianza y los valores promedios de las variables evaluadas se compilaron por tratamiento (Tabla 1).

\begin{tabular}{|c|c|c|}
\hline Variables & $\begin{array}{c}\text { Tratamiento } \\
\text { convencional }\end{array}$ & $\begin{array}{c}\text { Tratamiento } \\
\text { limpio }\end{array}$ \\
\hline $\begin{array}{c}\text { Número de tubérculos } \\
\text { por planta }\end{array}$ & 32,78 & 34,15 \\
\hline $\begin{array}{c}\text { Peso de tubérculos por } \\
\text { planta (g) }\end{array}$ & 31,91 & 36,28 \\
\hline $\begin{array}{c}\text { Tamaño de tubérculos } \\
\text { por planta (cm) }\end{array}$ & 10,86 & 11,06 \\
\hline $\begin{array}{c}\text { Peso total de los } \\
\text { tubérculos producidos } \\
\text { por planta (gr.) }\end{array}$ & 958,82 & 1028 \\
\hline
\end{tabular}

Tabla 1. Valores promedio de las variables evaluadas en los tratamientos limpio y convencional. Fuente: Los Autores. 2010.

\section{Número de tubérculos por planta}

En esta variable, el análisis no presentó diferencias estadísticamente significativas entre tratamientos limpio y convencional (Gráfica 1), aunque el tratamiento de producción limpia mostró el mayor número de tubérculos en promedio con 34.15 por planta. El número de tubérculos es una variable escasamente evaluada en trabajos de investigación, debido a que éstos se relacionan en la producción total del cultivo. Sin embargo, la literatura en general estima que una planta de papa criolla produce en promedio 40 tubérculos de diferentes tamaños y calidad.

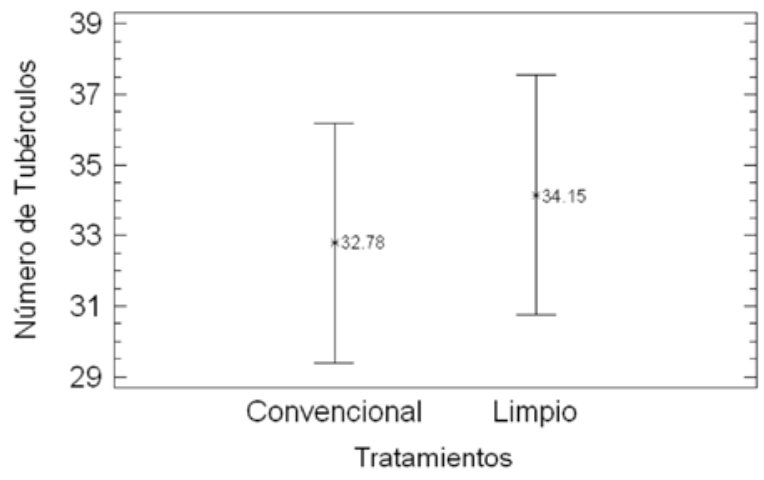

Gráfica 1. Efecto de los tratamientos en el número de tubérculos por planta. Fuente: Los autores. 2010 


\section{Tamaño y peso por tubérculo}

En tamaño y peso por tubérculo no se encontraron diferencias estadísticamente significativas entre los tratamientos. En la producción limpia se obtuvo un peso y longitud promedio de $36.35 \mathrm{~g}$ y $11.5 \mathrm{~cm}$ respectivamente, y en la producción convencional se obtuvo un peso y longitud promedio $31.87 \mathrm{~g}$ y 10.87 $\mathrm{cm}$ respectivamente (Gráficas 2 y 3 ). El comportamiento de la planta para producir tubérculos de características similares demuestra que la implementación de estrategias de manejo limpias pudo generar producciones con calidad en tamaño y peso similares a las de sistemas convencionales en el ambiente del Ceagro San Pablo.

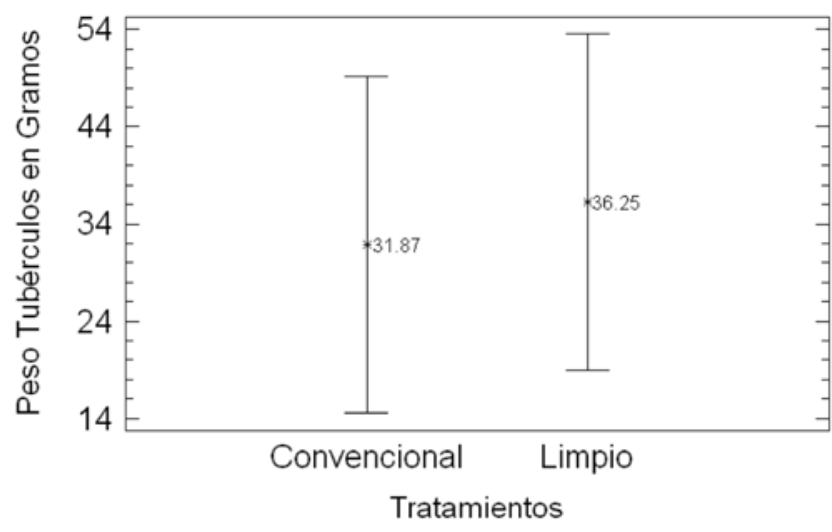

Gráfica 2. Efecto de los tratamientos en el peso promedio individual de los tubérculos. Fuente: Los autores. 2010

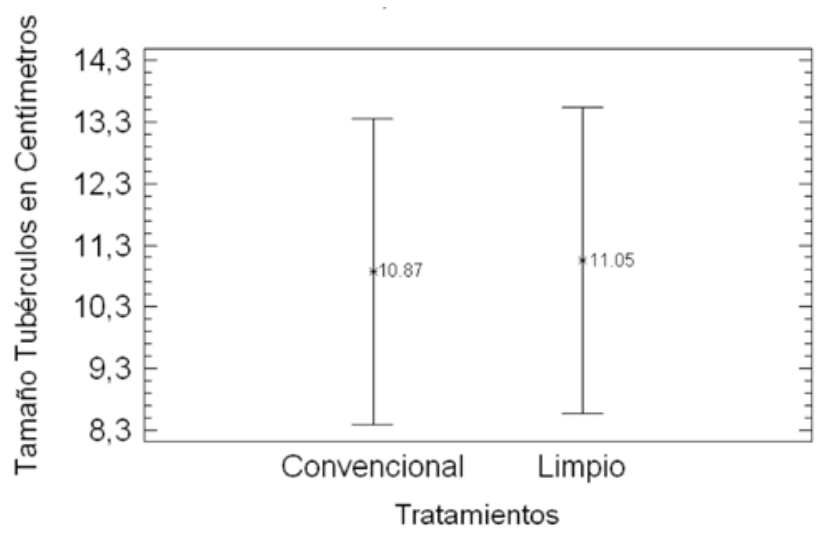

Gráfica 3. Efecto de los tratamientos en el tamaño individual de los tubérculos. Fuente: Los autores. 2010

\section{Peso de la producción por planta}

Esta variable evidenció que no se presentaron diferencias significativas entre tratamientos. En la producción limpia se obtuvo en promedio $1029.82 \mathrm{~g}$ de papa por planta y en la producción convencional se obtuvo $950.52 \mathrm{~g}$ de papa por planta (Gráfica 4). El peso total de la producción de tubérculos por planta con características comerciales, se considera como la variable más importante debido a que se muestra el potencial de producción del cultivo, reafirmando la validez de la implementación de estrategias de manejo limpio para el sistema productivo de papa criolla. El resultado en esta variable concuerda con lo obtenido para el número de tubérculos por planta, peso y tamaño. El sistema de fertilización implementado en la investigación que consistió en fraccionar la aplicación de los insumos en siembra y aporque, pudo influenciar en los rendimientos ya que Pérez et al. (2008), afirman que es más eficiente realizar una sola aplicación de fertilizante en siembra, teniendo en cuenta que la planta hace mejor uso de los nutrientes en los primeros días del establecimiento y el ciclo corto de la papa criolla (Figura 3).

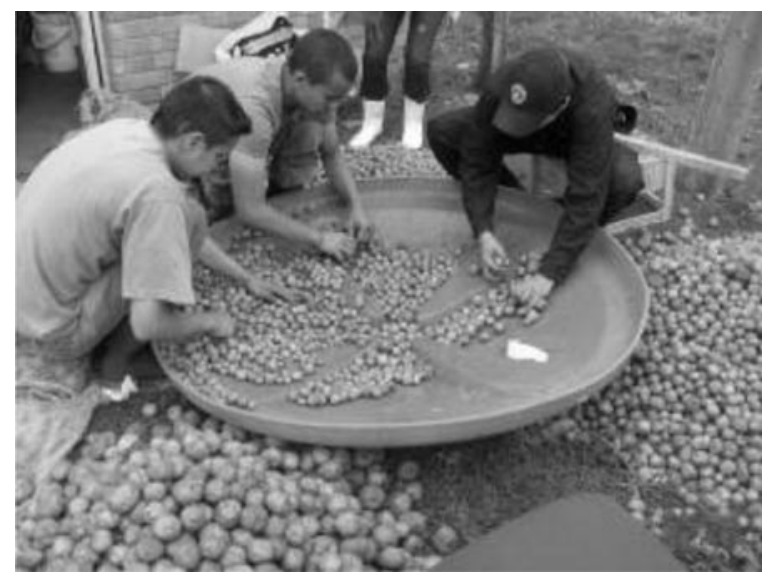

Figura 3. Cosecha y medición de variables. Fuente: Los autores. 2010.

Los resultados generales de la investigación también concuerdan con lo encontrado por Muñoz y Lucero (2008), quienes afirman que la fertilización química en papa criolla con altos volúmenes no aumenta los rendimientos frente a la fertilización limpia, siendo mas recomendable combinar la fertilización química y orgánica en una relación de 1:2.6 respectivamente. Los resultados también evidencian que el manejo de riesgos fitosanitarios mediante la implementación de estrategias limpias, produce mejores resultados que con el uso de plaguicidas de síntesis química. Esto en el ámbito ambiental y económico debido a que la presencia de plagas y enfermedades no influyó en la producción limpia.

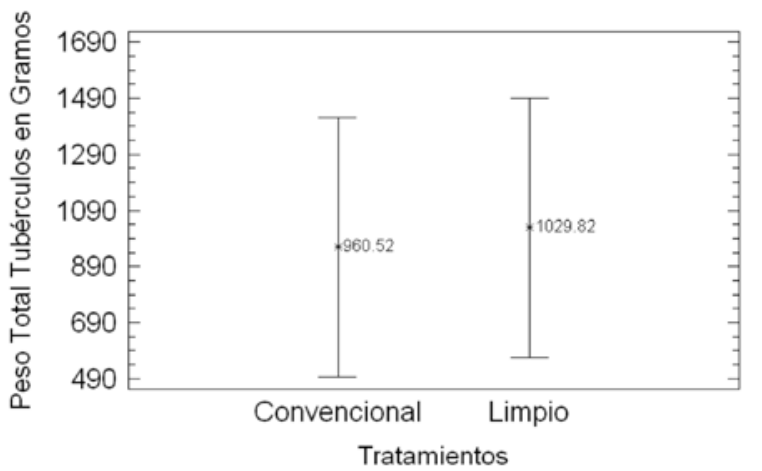

Gráfica 4. Efecto de los tratamientos en peso total de los tubérculos producidos por planta. Fuente: Los autores. 2010 


\section{Conclusiones}

- En las condiciones experimentales de este trabajo no se obtuvieron diferencias estadísticamente significativas en cuanto a las variables evaluadas: número, tamaño y peso de los tubérculos por planta y su producción total para el manejo limpio y convencional del sistema productivo de papa criolla.

- Lo anterior sugiere que, mediante la implementación de estrategias de producción limpia, se pueden obtener rendimientos similares a los generados en cultivos bajo técnicas de producción convencionales.

- Los resultados tienen importancia económica, social y ambiental en tanto que se puede sugerir al productor la supresión de la mayoría de insumos de síntesis química, de los cuales un gran porcentaje son persistentes en el ambiente. Para continuar con el proceso de la estandarización de metodologías para la producción limpia de papa criolla en el Ceagro San pablo, es recomendable continuar con trabajos que se concentren en el análisis económico para determinar la rentabilidad de la producción limpia.

\section{Referencias}

[1] Agencia de Noticias de Ciencia y Tecnología de Colombia (2005), Boletín: Colombianos producen tres nuevas variedades de papa criolla con gran potencial de exportación, Enero 19 de 2005.

[2] Arias V. (1996), "Evaluación del rendimiento en papa criolla (Solanum phureja) variedad "yema de huevo", bajo diferentes densidades de siembra en la sabana de Bogotá", en Agronomía Colombiana. 13(2), pp. 152-161.

[3] Buitrago G.; López A.; Coronado A. \& Osorno F. (2004) "Determinación de las características físicas y propiedades mecánicas de papa cultivada en CoIombia", en Revista Brasileira de Engenharia Agrícola e Ambiental, 8(1), pp. 102-110.

[4] Cevipapa (2004), Centro Virtual de Investigación de la Cadena Agroeclimentaria de la Papa. El cultivo de la papa en Colombia, disponible en: http://www. cevipapa.org.co/cultivo/colombia.php, recuperado: 30 de noviembre de 2009

[5] CIP, (1992), Control biológico de la polilla de la papa con Baculovirus phthorimaea, Boletín de Capacitación No. 2.

[6] Fedepapa (2005), Guía para el cultivo de papa, Panamericana Formas e Impresos S.A.

[7] Ministerio de Agricultura (2008), Área y Producción Agrícola y Pecuaria. Análisis - Estadísticas, disponible en: http://www.agronet.gov.co/, recuperado: 28 de noviembre de 2009.

[8] Ministerio de Agricultura (2009), Sistema de Información de Precios de Insumos y Factores, Ministerio de Agricultura y Desarrollo Rural - Corporación Colombia Internacional.

[9] Muñoz L. y Lucero M. (2008), "Efecto de la fertilización orgánica en el cultivo de papa criolla So/anum phureja", en Agronomía Colombiana, 26(2), pp. 340-346.

[10] Pérez L.; Rodríguez L. y Gómez M. (2003), "Efecto del fraccionamiento de la fertilización con $N, P, K$ y Mg y la aplicación de los micronutrientes $B$, Mn y Zn en el rendimiento y calidad de papa criolla (Solanum phureja) variedad Criolla Colombia", en Agronomía Colombiana, 26(3), pp. 477-486.

Maikol Santamaría Galindo Ingeniero en Agroecología. Docente Corporación Universitaria Minuto de Dios (UNIMINUTO). Facultad de Ingeniería. Programa de Ingeniería Agroecológica. Calle 81B \# $72 B$ 70. biologicalcontrol@gmail.com.

Johana Montañéz Gómez Estudiante Corporación Universitaria Minuto de Dios. Facultad de Ingeniería. Programa de Ingeniería Agroecológica. Calle 81B \# 72B 70 - johanamontanez@gmail.com

Rafael Ricardo Sánchez Polo Estudiante Corporación Universitaria Minuto de Dios. Facultad de Ingeniería. Programa de Ingeniería Agroecológica. Calle 81B \# 72B 70 - sanchez.polo@hotmail.com 